\title{
Resultados da correção cirúrgica da exotropia permanente em pacientes amblíopes e não-amblíopes
}

\author{
Surgical correction of permanent exotropia outcomes in amblyopic and non-amblyopic patients
}

Adriana Valim Portes ${ }^{1}$, Andrea Marcia B. V. Franco ${ }^{1}$, Marcela F. Tavares ${ }^{1}$, Carlos Ramos Souza-Dias ${ }^{1}$, Mauro Goldchmit ${ }^{2}$

\section{RESUMO}

A exotropia permanente (XT) acomete cerca de 1 a 2\% da população. Seu tratamento é clínico: antiambliogênico e correção dos erros refrativos, e cirúrgico. O objetivo do tratamento cirúrgico é alinhar os olhos na posição primária do olhar, proporcionando melhor resultado estético. Há muito tempo diversos autores estudam os fatores pré, per e pós-operatórios relacionados ao resultado cirúrgico, uma vez que a taxa de sucesso varia de 60 a $80 \%$. Ainda são poucos os estudos que comparam a presença de ambliopia como fator de influência no resultado final.

Objetivo: Comparar o resultado cirúrgico dos pacientes amblíopes e não-amblíopes submetidos à cirurgia de correção de XT.

Métodos: Análise retrospectiva de 37 prontuários de pacientes amblíopes (Grupo A) e não-amblíopes (Grupo B) submetidos à correção cirúrgica de XT por retrocessoressecção monocular, sendo avaliados os registros pós-operatórios imediatos e tardios. Idade: grupo A 24,7 $\pm 14,2$ anos, grupo B 22,6 $\pm 18,6$ anos; Desvio pré-operatório: grupo A $29,1 \pm 7,2^{\Delta}$, grupo B $28,4 \pm 6,8^{\Delta}$.

Resultados: A taxa de sucesso foi de $60 \%$ e $100 \%$ ( $p<0,05)$, no pós-operatório imediato e $50 \%$ e $82,3 \%$ ( $p=0,082)$, no pós-operatório final, nos grupos $A$ e $B$, respectivamente. Não houve diferença significante quanto aos desvios pós-operatórios imediatos, tardios e variação do desvio.

Conclusão: Pode-se concluir que o grupo B mostrou melhor resultado no pós-operatório imediato; porém não houve diferença no resultado cirúrgico de correção de exotropia permanente entre pacientes amblíopes e não-amblíopes no período pós-operatório de seis meses.

Descritores: Exotropia/cirurgia; Ambliopia

\begin{abstract}
Permanent exotropia (XT) occurs in 1 to $2 \%$ of the pediatric population. Its management involves careful assessment of patient, treatment of amblyopia, refractive errors and surgery. The aim of the surgery is to straighten the eyes in the primary gaze position, giving a better cosmetic outcome. The factors reported to affect surgical outcome after exotropia surgery vary widely in reports and success rates for strabismus surgery have been reported to range from $60 \%$ to $80 \%$. There are few reports to determine the relation between amblyopia and surgical outcome in exotropic patients.

Purpose: To compare the surgical outcome of permanent exotropia surgery in amblyopic and non-amblyopic patients.

Methods: This is a retrospective study of 37 clinical records from amblyopic patients (Group A) and non-amblyopic patients (Group B) who underwent recess-resect in one eye for XT. Postoperative deviation was analyzed in one month (immediate) and in six months (final) in both groups and in between. Age: group A $24.7 \pm 14.2$ years, group B $22.6 \pm 18.6$ years; Preoperative deviation: group A $29.1 \pm 7.2^{4}$, group B $28.4 \pm 6.8^{4}$.

Results: The success rate in the imediate postoperative period was 60\% (Group A) and $100 \%$ (Group B) ( $p<0.05$ ); 50\% (Group A) and $82.3 \%$ (Group B) ( $p=0.082$ ) in the final postoperative period. There was a statistical difference in the imediate postoperative deviation, but the final deviation and the variation of the deviation were similar in both groups.

Conclusion: There is a better outcome in patients of group B and no diference in the surgical outcome between these amblyopic and non-amblyopic patients in the final
\end{abstract} postoperative period.

Keywords: Exotropia/surgery; Amblyopia

\section{INTRODUÇÃO}

A exotropia permanente (XT) acomete de 1 a 2\% da população pediátrica ${ }^{(1,2)}$. Seu tratamento resume-se na eventual diminuição da ambliopia, correção de erros refrativos e a cirurgia. A taxa de sucesso do resultado cirúrgico varia entre 30 e $80 \%{ }^{(1)}$. O benefício sensorial que se pode oferecer aos portadores de XT é a cura ou a redução da ambliopia ${ }^{(3)}$. O objetivo do tratamento cirúrgico é alinhar os olhos na posição primária do olhar, fornecendo resultado estético ${ }^{(3,4)}$. Não é possível restabelecer visão binocular nas $X T^{(1)}$.

Kampanartsanyakorn et al. estudaram retrospectivamente 117 prontuários de pacientes portadores de exotropia essencial (15 a $50^{\Delta}$ ) que foram submetidos à primeira correção cirúrgica de estrabismo e encontrou taxa de sucesso de 57\%, sendo os melhores resultados alcançados nos pacientes com desvios pré-operatórios inferiores a $30^{\Delta}(p<0,001)$ e operados antes dos 6 anos de idade $(p<0,05)^{(1)}$. Hunter et al. estudaram 6 pacientes portadores de XT no primeiro ano de vida, que foram submetidos à correção cirúrgica, e obtiveram taxa de sucesso de 83\% (5 pacientes) em um ano de acompanhamento(5). Biglan et al. estudaram 8 pacientes portadores de XT submetidos à correção cirúrgica, com taxa de sucesso de 75\% (2 pacientes reoperados). Esses autores observaram que ortotropia nas primeiras avaliações pós-cirúrgicas é um fator predisponente à estabilidade do alinhamento ocular ${ }^{(6)}$.

Os fatores preditivos relacionados ao resultado cirúrgico variam na literatura e ainda não há consenso quanto a eles ${ }^{(7)}$. Scott et al. estudaram em 69 pacientes portadores de XT o resultado e a sua estabilidade pós-cirúrgica. Os desvios oculares desses pacientes variaram entre 20 e $80^{\Delta}$. Utilizou-se retrocesso bilateral dos retos laterais, retrocesso-ressecção unilateral ou operação de 3 ou 4 músculos horizontais como forma de corrigir os desvios oculares.

${ }^{1}$ Physician, Ophthalmology Department, Santa Casa de Misericórdia de São Paulo - SCMSP - São Paulo (SP), Brazil.

Physician, Ophthalmology Department, Santa Casa de Misericórdia de São Paulo - SCMSP - São Paulo (SP), Brazil; Strabismus Sector, CEMA Hospital - São Paulo (SP), Brazil.

Funding: No specific financial support was available for this study Disclosure of potential conflicts of interest: A.V.Portes, None; A.M.B.V.Franco, None; M.F.Tavares, None; C.R.Souza-Dias, None; M.Goldchmit, None.

Correspondence address: Adriana Valim Portes. Rua Conselheiro Brotero - 823 Ap. 132 - São Paulo (SP) - 01232-011 - Brazil -E-mail: dri.portes@gmail.com 
Dos pacientes que apresentaram supercorreção inicial de $15^{\Delta}$ ou mais, $70 \%$ permaneceram com desvios de até $9^{\Delta}$ ao final de 2 anos. Dos supercorrigidos em 4 a 14 $4^{\Delta}, 81 \%$ permaneceram com desvios de até $9^{\Delta}$ neste mesmo período. Dos pacientes que estavam em ortotropia no período pós-operatório imediato e dos que ficaram subcorrigidos (4 a $14^{\Delta}$ ), apenas $69 \%$ e 50\%, respectivamente, mantiveram o desvio de até $9^{\Delta}$ nos próximos 2 anos. Portanto, nesse estudo, supercorreção entre 4 e $14^{\Delta}$ na avaliação pós-cirúrgica inicial foi o fator relacionado ao melhor alinhamento ocular entre 1 e 2 anos após o procedimento(8).

Ainda são poucos os estudos que comparam a presença ou não de ambliopia como fator de influência no resultado final. Este estudo teve como objetivo comparar o resultado cirúrgico dos pacientes amblíopes e não-amblíopes submetidos à cirurgia corretora de exotropia permanente.

\section{MÉTODOS}

Foram examinados retrospectivamente 37 prontuários de pacientes portadores de XT oriundos da seção de Motilidade Ocular Extrínseca do Departamento de Oftalmologia da Santa Casa de Misericórdia de São Paulo, do setor de Estrabismo do Instituto CEMA e das clínicas particulares do Dr. Carlos Ramos Souza-Dias e Dr. Mauro Goldchmit. Esses pacientes foram submetidos à correção cirúrgica com retrocesso-ressecção monocular no período compreendido entre 2001 e 2008. Os pacientes foram separados em dois grupos, a saber: grupo A - pacientes amblíopes e grupo B - pacientes nãoamblíopes. Foram incluídos pacientes cujo desvio pré-operatório variou entre 20 e 40 ${ }^{4}$, sem visão binocular, sem acometimento dos músculos retos verticais ou oblíquos e que mantiveram acompanhamento pós-cirúrgico por no mínimo seis meses. Foram excluídos os prontuários de pacientes com diagnóstico de exotropia intermitente, exotropia consecutiva ou que já haviam sido submetidos a cirurgias para correção de estrabismo.

Todos os pacientes foram submetidos a exame oftalmológico, que incluiu medida da acuidade visual (tabela de Snellen ou cartões de Teller), refratometria sob cicloplegia, biomicroscopia e fundoscopia. Foi considerada ambliopia a diferença maior ou igual a duas linhas de visão na tabela de Snellen entre os olhos. Os valores das acuidades visuais foram convertidos para logMAR. A medida do desvio foi feita com os testes de cobertura alternado com prisma ou Krimsky, quando a acuidade visual ou a colaboração do paciente não permitiu a realização do primeiro teste. Para o registro dos dados, foram adotados valores positivos para os desvios divergentes e valores negativos para os convergentes.

O grupo A apresentou acuidade visual de movimento de mãos a 0,1 logMAR no olho com pior visão e no olho de melhor visão a acuidade variou entre 0,1 a 0 logMAR. O grupo B apresentou acuidade visual de no mínimo 0,5 logMAR, em ambos os olhos.

As informações relativas ao sexo, idade e desvio pré-operatório encontram-se na tabela 1.

Nos pacientes do grupo A, a cirurgia foi realizada no olho amblíope e no grupo B no olho não dominante. Os planos cirúrgicos foram calculados de acordo com a magnitude do desvio ocular.

Considerou-se como desvio pós-operatório imediato a medida do desvio ocular registrado entre o primeiro dia e o primeiro mês pós-operatório, desvio pós-operatório final aquele medido com seis meses após a cirurgia e a variação do desvio a diferença observada entre as duas medidas.

Os resultados foram classificados em bom (ortotropia $\pm 10^{\Delta}$ ) e mau (exotropia ou esotropia $>10^{\Delta}$ ), no período pós-operatório imediato

A análise dos resultados obtidos foi realizada adotando-se nível de significância de $5 \%(p \leq 0,05)$. Todos os dados obtidos foram expressos pelo valor médio, desvio e erro padrão e foram analisados usando o programa estatístico SigmaStat for Windows (versão 1.0; Jandel
Tabela 1. Distribuição segundo o sexo, a idade e o desvio pré-operatório dos pacientes com exotropia permanente ambliopia (Grupo A) e exotropia permanente e sem ambliopia (Grupo B) submetidos à correção cirúrgica monocular

\begin{tabular}{|c|c|c|}
\hline Amostra & $\begin{array}{c}\text { Grupo A } \\
\text { Amblíopes }(n=20)\end{array}$ & $\begin{array}{c}\text { Grupo B } \\
\text { Não-amblíopes }(n=17)\end{array}$ \\
\hline $\begin{array}{l}\text { Sexo (F/M) (\%) } \\
\text { Idade (anos) } \\
\text { Desvio pré-operatório }(\Delta)\end{array}$ & $\begin{array}{c}45 / 55 \\
24,7 \pm 14,2(6 \text { a } 48) \\
29,1 \pm 7,2(20 \text { a } 40)\end{array}$ & $\begin{array}{c}29 / 71 \\
22,6 \pm 18,6(1 \text { a } 79) \\
28,4 \pm 6,8(20 \text { a } 40)\end{array}$ \\
\hline
\end{tabular}

Scientific Software, San Jose, California, EUA). Os testes utilizados foram: testes $t$ pareado e de Student; teste de Mann-Whitnney; teste exato de Fisher e análise de variância (Kruskal-Wallis One Way Analysis of Variance on Ranks).

Este trabalho foi aprovado pelo Comitê de Ética em Pesquisa da Santa Casa de Misericórdia de São Paulo (número: 230/09).

\section{RESULTADOS}

Os resultados encontram-se nas tabelas 2 a 4 e no gráfico 1.

\section{COMENTÁRIOS}

Teoricamente, o desvio pré-cirúrgico é o mesmo antes e após anestesia com relaxante muscular (pancurônio). Assim, retrocessoressecção de um defeito mecânico aparente unilateral tem grande chance de resultar em alinhamento ocular estável. Portanto, a correção cirúrgica pode ser realizada apenas no olho desviado. Entretanto, em alguns casos, observa-se desvio do outro olho sob anestesia geral e, assim, esse deve ser considerado no plano cirúrgico a ser realizado ${ }^{(9)}$. Como este trabalho foi um estudo retrospectivo os planos aqui relatados já foram os considerados de acordo com a posição dos olhos sob anestesia geral.

A tabela 2 evidencia diferença estatisticamente significante entre os desvios pré-operatórios e pós-operatórios nos dois grupos de estudo ( $p<0,0001)$; entretanto, ao se comparar os desvios pósoperatórios de cada grupo, a diferença não foi estatisticamente significante.

Alguns autores classificam sucesso cirúrgico como ortotropia $\pm 8^{\Delta(10)}$ ortotropia $\pm 5^{\Delta(4)}$ ou ainda como desvio convergente menor que $6^{\Delta}$ ou divergente inferior a $11^{\Delta(7)}$. Para este estudo, foi adotado o critério de sucesso cirúrgico como ortotropia $\pm 10^{\Delta}$, assim como a maioria dos autores ${ }^{(1,11-14)}$

O sucesso desses procedimentos depende de avaliação pré-operatória completa, considerando-se os possíveis fatores desestabilizadores $^{(15)}$. Oh et al. não identificaram nenhum fator pré-operatório relacionado ao resultado cirúrgico e apenas o desvio pós-operatório inicial mostrou-se relevante para o resultado final ${ }^{(7)}$. Kushner et al. correlacionaram resultado final e comprimento axial, avaliando também a relação entre a idade e o erro refrativo. Identificaram melhores resultados nos pacientes operados antes da maturidade sensorial ${ }^{(16)}$. Outros autores também identificaram idade como fator relacionado ao sucesso cirúrgico, relatando melhores resultados nos pacientes operados antes dos seis anos de idade ${ }^{(1)}$. Entretanto, na maioria dos trabalhos encontrados, a variável mais relacionada ao sucesso cirúrgico foi uma supercorreção inicial $\left.\right|^{(7-9,13,17)}$

Como apresentado na tabela 3, os desvios pós-operatórios dos dois grupos mostraram-se semelhantes (desvio pós-operatório imediato: $p=0,228$, final: $p=0,143)$, com valores discretamente superiores, tanto para as médias quanto para os desvio-padrões, para o grupo A, mas ainda assim dentro do que consideramos sucesso cirúrgico. A variação dos desvios também mostrou maiores valores no grupo A, não havendo diferença estatisticamente significante entre os grupos estudados $(p=0,147)$. 
Tabela 2. Comparação entre desvios pré-operatórios, pós-operatórios imediatos e finais, nos pacientes com exotropia permanente e ambliopia (Grupo A) e nos pacientes com exotropia permanente e sem ambliopia (Grupo B)

\begin{tabular}{|c|c|c|c|c|c|}
\hline \multicolumn{3}{|c|}{$\begin{array}{c}\text { Grupo A } \\
\text { Desvios (A) }\end{array}$} & \multicolumn{3}{|c|}{$\begin{array}{c}\text { Grupo B } \\
\text { Desvios ( }(\Delta)\end{array}$} \\
\hline Pré-operatório & Pós-operatório imediato & Pós-operatório final & Pré-operatório & Pós-operatório imediato & Pós-operatório final \\
\hline $29,10 \pm 7,25$ & $\begin{array}{c}6,50 \pm 8,13 \\
I C 18,5-26,7 \\
p^{1}<0,0001^{*}\end{array}$ & $\begin{array}{c}9,15 \pm 10,15 \\
I C 14,4-25,5 \\
p^{2}<0,0001^{*} \\
p^{3}=0,3 * * \\
I C-8,5-3,2\end{array}$ & $28,41 \pm 6,77$ & $\begin{array}{c}2,18 \pm 3,07 \\
\text { IC } 21,9-30,6 \\
p^{\prime}<0,0001^{* * *}\end{array}$ & $\begin{array}{c}4,88 \pm 6,80 \\
\text { IC } 17,8-29,3 \\
\mathrm{p}^{2}<0,0001^{* * *} \\
\mathrm{p}^{3}=0,5009^{* * * *}\end{array}$ \\
\hline
\end{tabular}

Pós-operatório final= 6 meses; $\mathrm{p}^{1}=$ pré-operatório $\times$ pós-operatório imediato; $\mathrm{p}^{2}=$ pré-operatório $\times$ pós-operatório final; $\mathrm{p}^{3}=$ pós-operatório imediato $\times$ final; ${ }^{*}=$ teste t pareado; ${ }^{* *}=$ teste t Student; ${ }^{* *}=$ teste t pareado; ${ }^{* * *}=$ teste Mann-Whitney

Tabela 3. Distribuição segundo a magnitude do retrocesso e da ressecção, desvios pós-operatórios e variação dos desvios dos pacientes com exotropia permanente e ambliopia (Grupo A) e sem ambliopia (Grupo B) submetidos à correção cirúrgica monocular

\begin{tabular}{|c|c|c|c|c|c|}
\hline \multirow{2}{*}{$\begin{array}{l}\text { Variável analisada } \\
\text { Retrocesso RL (mm) }\end{array}$} & \multicolumn{2}{|c|}{ Grupo A } & \multicolumn{2}{|c|}{ Grupo B } & \\
\hline & $6,8 \pm 1,2$ & (5 a 9$)$ & $6,4 \pm 0,8$ & (5 a 8$)$ & \\
\hline Ressecção RM (mm) & $5,1 \pm 0,8$ & (4 a 7$)$ & $4,9 \pm 0,6$ & $(4$ a 6$)$ & \\
\hline Desvio imediato ${ }^{(\Delta)}$ & $6,5 \pm 8,1$ & $(-5$ a 20$)$ & $2,2 \pm 3,1$ & $(-2$ a 8$)$ & $p=0,228$ \\
\hline Desvio final( ${ }^{(\Delta)}$ & $9,1 \pm 10,0$ & $(-8$ a 35) & $4,9 \pm 6,8$ & (0 a 20) & $p=0,143$ \\
\hline Variação do desvio(s) & $5,0 \pm 5,6$ & (0 a 20) & $3,3 \pm 5,4$ & (0 a 17) & $p=0,147$ \\
\hline
\end{tabular}

Teste estatístico utilizado= Mann-Whitney; $\mathrm{RL}=$ reto lateral; $\mathrm{RM}=$ reto medial

Tabela 4. Distribuição dos casos de exotropia permanente submetidos à cirurgia monocular de acordo com o resultado cirúrgico

\begin{tabular}{lcccc}
\hline Momento da avaliação & Grupo & Bom & Mau & \\
\hline Pós-operatório imediato & A & $12(60,0 \%)$ & $8(40,0 \%)$ & $\mathrm{p}<0,05^{*}$ \\
& B & $17(100,0 \%)$ & 0 & \\
Pós-operatório final & A & $10(50,0 \%)$ & $10(50,0 \%)$ & $\mathrm{p}=0,082^{*}$ \\
& B & $14(82,3 \%)$ & $3(17,7 \%)$ & \\
\hline
\end{tabular}

$\mathrm{Bom}=\mathrm{OT} \pm 10^{\Delta} ; \mathrm{Mau}=\mathrm{ET}>10^{\Delta}$ ou XT $>10^{\Delta} ; \mathrm{A}=$ grupo com ambliopia; $\mathrm{B}=$ grupo sem ambliopia; ${ }^{*}=$ teste exato de Fisher

Como mostra a tabela 4, obtivemos bom resultado em 12 dos 20 pacientes do grupo A (60\%) e em todos os pacientes do grupo B (100\%) no período pós-operatório imediato, diferença estatisticamente significante $(p<0,05)$. Nosso resultado assemelha-se aos da literatura, que revela taxa de sucesso inicial variando de 60 a $80 \%^{(1-3,5)}$. Já no período pós-operatório final obtivemos 50\% (10 pacientes) de bom resultado no grupo A e 82,3\% (14 pacientes) no grupo B, diferença estatisticamente não significante $(p=0,082)$. Essa diferença é muito próxima da significância, mas não podemos afirmar que com o aumento da amostra nossos resultados seriam diferentes dos encontrados. Portanto, os pacientes amblíopes comportaram-se de maneira semelhante aos não-amblíopes, apesar de inicialmente terem tido taxas de sucesso diferentes. Tal fato pode também ser observado pela análise da variância dos desvios pós-operatórios, que não mostrou diferença significante (Gráfico 1).

Alguns autores mostraram que $80 \%$ dos desvios oculares pósoperatórios modificam-se nas primeiras oito semanas após a cirurgia ${ }^{(17)}$. Scott et al. relataram variação nas primeiras 6 semanas e posterior estabilidade por 2 anos. Identificaram também variação média de 6 a $11^{\Delta(8)}$. Neste estudo, pudemos observar que a variação média do desvio foi inferior à descrita na literatura: $5^{\Delta} \pm 5,6$ no grupo $A$ e $3,3^{\Delta} \pm 5,4$ no grupo B (Tabela 3 )

Encontram-se poucos trabalhos que exploram a relação entre ambliopia e resultado cirúrgico final. Nenhum desses mostrou relação significante entre essas características ${ }^{(4,14)}$. Abbasoglu et al. es-

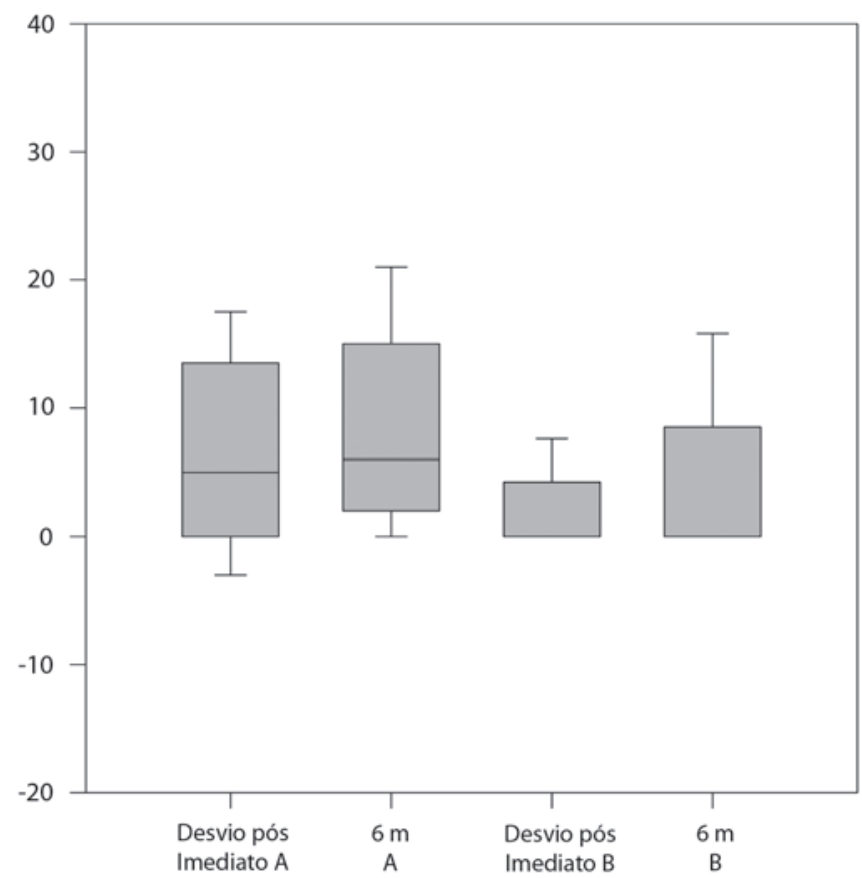

Kruskal-Wallis One Way Analysis of Variance on Ranks; $p=0,1041$; linhas horizontais - mediana e quartis

Gráfico 1. Análise de variância dos desvios pós-operatórios nos pacientes com exotropia permanente e ambliopia (Grupo A) e sem ambliopia (Grupo B) submetidos à correção cirúrgica monocular

tudaram diversas variáveis pré-operatórias possivelmente relacionadas ao resultado cirúrgico, não identificando ambliopia como fator relevante. Apenas o desvio pré-operatório mostrou diferença significante para os resultados finais, havendo melhores respostas para os maiores ângulos. Sugerem que sejam realizados estudos com maiores casuísticas e melhores avaliações pré e pós-operatórias 
para resultados mais confiáveis ${ }^{(4)}$. Gezer et al., também estudaram os fatores que interferem no resultado cirúrgico e, assim como os outros autores, não identificaram ambliopia como fator determinante do resultado cirúrgico(14). Neste estudo não houve diferença entre os dois grupos avaliados quanto ao desvio pós-operatório final e à variação do desvio pós-operatório, apesar de sucesso inicial superior do grupo dos pacientes não-amblíopes. A supressão nos portadores de XT é muito intensa, não importando se os pacientes são amblíopes ou não para o resultado final.

Muitos outros fatores pré, per e pós-operatórios podem estar implicados no resultado final da cirurgia, mas ainda faltam estudos com casuística adequada para determiná-los em pacientes portadores de exotropia permanente.

\section{CONCLUSÃO}

Pode-se concluir com o presente estudo que no período pósoperatório imediato o grupo dos pacientes não-amblíopes apresentou melhor resultado que o dos pacientes amblíopes. Entretanto, não houve diferença no resultado das cirurgias de correção de exotropia permanente entre os dois grupos estudados no final dos seis meses do estudo.

\section{REFERÊNCIAS}

Kampanartsanyakorn S, Surachatkumtonekul T, Dulayajinda D, Jumroendararasmee M, Tongsae S. The outcomes of horizontal strabismus surgery and influencing factors of the surgical success. J Med Assoc Thai. 2005:88 Suppl 9:S94-9.

2. Mohney BG, Huffaker RK. Common forms of childhood exotropia. Ophthalmology. 2003; 110(11):2093-6.
3. Díaz JP, Dias CS. Estrabismo. 4a ed. São Paulo: Santos Editora; 2002

4. Abbasoglu OE, Sener EC, Sanac AS. Factors influencing the successful outcome and response in strabismus surgery. Eye (Lond). 1996;10(Pt 3):315-20.

5. Hunter DG, Kelly JB, Buffenn AN, Ellis FJ. Long-term outcome of uncomplicated infantile exotropia. J AAPOS. 2001;5(6):352-6.

6. Biglan AW, Davis JS, Cheng KP, Pettapiece MC. Infantile exotropia. J Pediatr Ophthalmo Strabismus. 1996;33(2):79-84

7. Oh JY, Hwang JM. Survival analysis of 365 patients with exotropia after surgery. Eye (Lond). 2006;20(11):1268-72.

8. Scott WE, Keech R, Mash AJ. The postoperative results and stability of exodeviations. Arch Ophthalmol. 1981;99(10):1814-8.

9. Jampolsky A. Treatment of exodeviations. In: Crawford JS. Pediatric ophthalmology and strabismus: transactions of the New Orleans Academy of Ophthalmology. New York: Raven Press; 1986

10. Abroms AD, Mohney BG, Rush DP, Parks MM, Tong PY. Timely surgery in intermittent and constant exotropia for superior sensory outcome. Am J Ophthalmol. 2001;131(1):111-6.

11. Jeoung JW, Lee MJ, Hwang JM. Bilateral lateral rectus recession versus unilateral recessresect procedure for exotropia with a dominant eye. Am J Ophthalmol. 2006;141(4):683-8. Comment in Am J Ophthalmol. 2006;142(4):708-9; author reply 709

12. Aslanis D, Follidi V, Constantopoulos I, Spyropoulos G, Paikos P. Résultats chirurgicaux des exotropies primitives concmitantes à grand angle chez les enfants. J Fr Ophtalmol. 2006; 29(1):37-42.

13. Keenan JM, Willshaw HE. The outcome of strabismus surgery in childhood exotropia. Eye (Lond). 1994:8(Pt 6):632-7. Comment in Eye (Lond). 1996:10(Pt 1):151.

14. Gezer A, Sezen F, Nasri N, Gözüm N. Factors influencing the outcome of strabismus surgery in patients with exotropia. J AAPOS. 2004;8(1):56-60

15. Burian HM, Spivey BE. The surgical management of exodeviations. Am J Ophthalmol. 1965; 59:603-20.

16. Kushner BJ, Fisher MR, Lucchese NJ, Morton GV. Factors influencing response to strabismus surgery. Arch Ophthalmol. 1993;111(1):75-9. Comment in Arch Ophthalmol. 1993; 111(10):1312.

17. Raab EL, Parks MM. Recession of the lateral recti. Early and late postoperative alignments. Arch Ophthalmol. 1969;82(2):203-8.

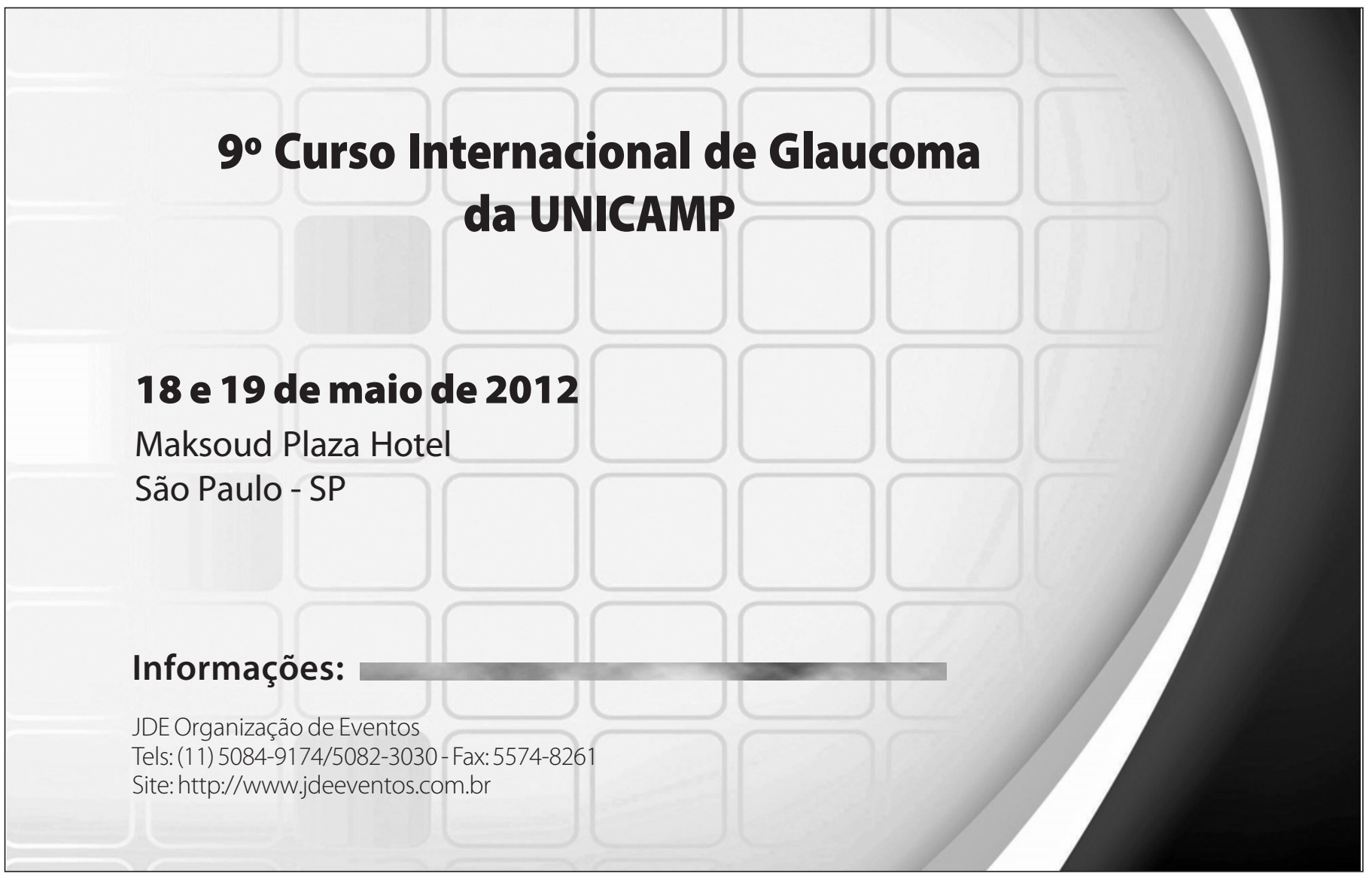

